\title{
Comparative analysis of nitrogen metabolism-related genes in two inbred maize lines with different low-nitrogen tolerance
}

\author{
M. YAN, J. LUO, L. LIANG, L. CHEN, Y.Y. CAO, Y.H. CHEN, X.Y. ZHU, and C.M. YU* \\ Scientific Observing and Experimental Station of Maize in Plains Area of Southern Region, \\ Ministry of Agriculture and School of Life Sciences, Nantong University, Nantong 226019, Jiangsu, P.R. China
}

\begin{abstract}
Maize is an important crop and its nitrogen use efficiency (NUE) has been an issue for plant scientists and breeders for many years. To meet the demand of genetic diversity in cultivating local elite maize cultivars, researchers need to isolate germplasms with a high NUE. In this study, 30 maize inbred lines were screened under hydroponic conditions, and three inbred lines that tolerate low nitrogen concentration were identified. A comparative analysis of genes involved in $\mathrm{N}$ uptake, transport, and assimilation between two lines with different tolerances revealed that the low-nitrogen-tolerant inbred line MBST exhibited higher expressions of nitrate and ammonium transporters, especially ZmNRT3.1B, than less tolerant B73. This suggests that the MBST line had a more efficient high-affinity transporter system. We also showed that lownitrogen conditions down-regulated the expressions of master genes, ZmNLPs, which were negatively correlated with the expressions of the nitrate transporters $Z m N R T s$. This indicates the existence of regulators that antagonize the function of ZmNLPs. Some genes related to N assimilation and carbon metabolism were also more expressed in MBST. This research shows that the low-nitrogen-tolerant line, MBST, transported nitrate and ammonium more effectively than the B73 line. The NUE was also higher in MBST than in B73.
\end{abstract}

Additional key words: gene expression, high-affinity transporter, nitrogen use efficiency, Zea mays.

\section{Introduction}

Inorganic nitrogen fertilizers are often applied to the soil for crop yield improvement. However, research data has shown that only about half of the amount applied to the soil is absorbed by plants, whereas the remaining half is washed into rivers and lakes, resulting in water pollution and leading to great pressure on the environment. Hence, cultivating crop cultivars with high nitrogen use efficiency (NUE) can decrease the need for fertilizer usage and benefit both farmers and the environment (Guan 2017, Li et al. 2017).
Maize is the most important crop in world. The mechanisms of the uptake and utilization of nitrate and ammonium in maize have been widely studied. Due to the correlation between $\mathrm{N}$ fertilizer applied to the soil and the crop yield, scientists in China have divided high NUE maize cultivars into two major types (Shen and Wang 2016): those with high $\mathrm{N}$ absorption efficiency and high yield under conditions of low $\mathrm{N}$ input and those whose yield increases only with an increase in $\mathrm{N}$ fertilizer application. Undoubtedly, the latter type will result in an increase in the release of nitrogen to the environment, causing severe environmental pollution. Therefore, it

Submitted 9 November 2018, last revision 16 May 2019, accepted 19 May 2019.

Abbreviations: AMT - ammonium transporter; CK - control; CPK - $\mathrm{Ca}^{2+}$-sensor protein kinases; CR - crown root; FENR - ferredoxinNADP reductase; GARP domain - a single Myb-related DNA-binding domain, which was named using the initials of the GARP domaincontaining proteins GOLDEN2, ARR-B, and Psr1; Gln - glutamine; Glu - glutamic acid; GPDH - glucose-6-phosphate dehydrogenase; GPI - glucose-6-phosphate isomerase; GPT - glucose-6-phosphate translocator; GS - glutamine synthetase; HATS - high-affinity transport system; HRS - hypersensitive to low Pi-elicited primary root shortening; LATS - low-afinity transport system; LBD - lateral organ boundary domain; LN - low nitrate; NIGT - nitrate inducible GARP-type transcriptional repressor; NIR - nitrite reductase; NLP - nodule inception-like protein; NPF - nitrate transporter/peptide transporter family; NR - nitrate reductase, NRE - nitrate-responsive cis-element; NRT - nitrate transporter; NUE - nitrogen utilization efficiency; PR - primary root; SNP - single nucleotide polymorphisms; SR - seminal root; TF - transcription factor.

Acknowledgments: This research was supported by the National Program on Key Basic Research Project (2016YFD0100500); the Technology Fund of Nantong City (MS12017022-7); the Natural Science Foundation of Jiangsu Province, China (Grant No. BK20151269), the Provincial Key Laboratory of Agrobiology, Jiangsu Academy of Agricultural Sciences (4911404202015K002); and the Large Instruments Open Foundation of Nantong University (KFJN1822). We thank a postgraduate student Lingjuan Wang who assisted to propagate maize seeds.

* Corresponding author; e-mail: ychmei@ntu.edu.cn 
is very important to select cultivars that have high NUE under low nitrogen conditions. Some inbred lines with high NUE have been developed, including ZD958, Ye478, and Gaspe Flint (Wang et al. 2005, Garnett et al. 2013, Han et al. 2015). However, to meet the demand of genetic diversity in local elite maize cultivars, researchers need to isolate more germplasms with high NUE.

In recent decades, some of the genes involved in $\mathrm{NO}_{3}{ }^{-}$ and $\mathrm{NH}_{4}{ }^{+}$transport and assimilation have been identified. For example, there are 53 members of the nitrate transporter/peptide transporter family (NRTs/NPFs) in Arabidopsis, 93 in rice, and 97 in maize (Plett et al. 2010, Léran et al. 2014, Guan 2017, Li et al. 2017). So far, only a few of these have been well elucidated in planta. According to the affinity of NRTs/NPFs for nitrate, they have been divided into two subclasses, namely high-affinity transport systems (HATS) and low-affinity transport systems (LATS). Generally, members of the gene family of nitrate transporter 2 (NRT2s) are classified as HATS, functioning at low environmental nitrate concentrations, whereas NRT1 family members are classified as LATS, functioning at high nitrate concentrations (Garnett et al. 2013, Li et al. 2017, Guan 2017). Interestingly, some NRT1s, such as AtNRT1.1 in Arabidopsis, serve as dualaffinity transporters, involved in both HATS and LATS (Liu and Tsay 2003). An ortholog of AtNRT1.1 in maize, ZmNRT1.1B, is a pH-dependent monophasic high-affinity nitrate transporter (Wen et al. 2017). Many NRT2 family members require nitrate assimilation-related protein (NAR, a respective gene which belongs to the NRT3 gene family), as a partner to transport nitrate (Laugier et al. 2012, Li et al. 2017, Kiba et al. 2018). When $\mathrm{NO}_{3}{ }^{-}$is transported in plants, it is further reduced to nitrite $\left(\mathrm{NO}_{2}^{-}\right)$by nitrate reductase (NR) and then to $\mathrm{NH}_{4}{ }^{+}$by nitrite reductase (NIR). Plants acquire environmental $\mathrm{NH}_{4}{ }^{+}$through ammonium transporters (AMTs). Inorganic ammonium is converted into its organic form by two enzymes, glutamine synthetase (GS) and glutamate synthase (GOGAT) through the GSGOGAT cycle (Li et al. 2017). Proteins, nucleic acid, and other N-containing compounds usually obtain elemental $\mathrm{N}$ through glutamic acid (Glu) and glutamine (Gln; Plett et al. 2010, Guan 2017, Li et al. 2017). In addition to $\mathrm{NO}_{3}^{-}$ and $\mathrm{NH}_{4}{ }^{+}$transporters, urea transporters are also present in plants. For example, $Z m D U R 3$ is a high-affinity urea transporter that can functionally complement yeast $d u r 3$ and Arabidopsis Atdur3 mutants, which also respond to nitrate depletion, but not to urea, suggesting that it is regulated by the nitrate signaling pathway (Zanin et al. 2014, Liu et al. 2015).

In recent years, some regulators controlling the transcription of NRTs and AMTs have been functionally characterized in Arabidopsis. For example, it has been shown, that after long-term nitrogen starvation resupplying nitrate to the medium evokes high $\mathrm{Ca}^{2+}$ content in the cytoplasm, which triggers the activity of $\mathrm{Ca}^{2+}$-sensor protein kinases (CPKs) to phosphorylate AtNLP. Phosphorylated AtNLP then enters the nucleus to induce the expression of downstream genes, such as AtNRT, AtNR, and AtNIR (Castaings et al. 2009, Konishi and Yanagisawa 2013, Yan et al. 2016, Liu et al. 2017). Very recently, ZmNLPs were identified in maize and their biological roles were verified in Arabidopsis Atnlp 7-4 mutants (Cao et al. 2017, Wang et al. 2018), but the target genes regulated by $Z m N L P s$ are not yet known. Other transcriptional regulators are lateral organ boundary domain (LBD) and nitrate-inducible GARP-type transcriptional repressor 1 (AtNIGT1). The GARP proteins have roles as repressors to down-regulate N-responsive genes, such as AtNRT2.1 and AtNRT2.4, under high nitrate concentrations (Riechann et al. 2000, Kiba et al. 2018, Maeda et al. 2018). Plant hormones also cooperate with $\mathrm{N}$ signals to harmonize plant growth (Medici and Krouk 2014, Guan 2017). Regrettably, there has been little research on these master genes in maize. Further research is required to determine whether different classes of plants (monocot vs. dicots) share common nitrogen signalling mechanisms.

Transcriptome data from maize has shown that perturbing nitrogen input influences the expression of many genes. Not only the genes of $\mathrm{N}$ transport and assimilation mentioned above, but also genes involved in carbon metabolism and stress defense, as well as long non-coding RNAs (Lv et al. 2016, Nazir et al. 2016). Although many genes have exhibited up- or down-regulated expression patterns, the results usually depend on the genotype of the maize being studied. To identify conserved genes that confer resistance to nitrogen stress, gene expression studies need to be performed more broadly, in different genotypes and especially in those with low nitrogen tolerance.

In the current study, we firstly screened low-nitrogen (LN)-tolerant inbred lines grown in hydroponic culture. We then utilized quantitative PCR to detect changes in the transcription of ZmNRT/NPFs, ZmATMs, ZmNLP, $\mathrm{N}$ assimilation-related genes, carbon metabolism-related genes, and some other genes in two cultivars (B73 and MBST) with different LN tolerance. Our aims were to determine: 1) how these genes responded to LN, 2) the relationship between $Z m N L P s$ and $Z m N R T / N P F$ s, and 3) the association between gene expression and the tolerance of maize to LN. This research can not only provide LNtolerant germplasm resources, but also increase our theoretical knowledge of LN tolerance in maize.

\section{Materials and methods}

Plants and treatments: Seeds of 30 inbred maize (Zea mays L.) lines (Table 1 Suppl.) were surface sterilized by $3 \%(\mathrm{v} / \mathrm{v}) \mathrm{H}_{2} \mathrm{O}_{2}$ for $10 \mathrm{~min}$, washed five times with sterilized distilled water before sowing on moist filter paper. The seeds were incubated in darkness at $22-24{ }^{\circ} \mathrm{C}$ for $3 \mathrm{~d}$. Then seedlings were transferred into planting baskets (55 $\mathrm{mm}$ diameter, 2 plants per each baskets) to grow for 7 - 8 days in climate chamber with a 14-h photoperiod, day/night temperatures of $22 / 20{ }^{\circ} \mathrm{C}$, and an irradiance of about $300 \mu \mathrm{mol} \mathrm{m} \mathrm{m}^{-2} \mathrm{~s}^{-1}$. Before treatment, the remaining tissue of seeds were removed to make sure that the nutrients were absorbed only through roots. Then, similar seedlings were separated into two groups for different treatments. Two different $\mathrm{N}$ concentrations were used: normal $\mathrm{N}$ supply $(\mathrm{CK})$ containing $1 \mathrm{mM} \mathrm{NH}_{4} \mathrm{NO}_{3}$ 
$+5 \mathrm{mM} \mathrm{KNO}_{3}(6 \mathrm{mM}$ nitrate $+1 \mathrm{mM}$ ammonium $)$ and low nitrogen supply (LN) containing $0.5 \mathrm{mM} \mathrm{NH}_{4} \mathrm{NO}_{3}$ $+0.5 \mathrm{mM} \mathrm{NH}_{4} \mathrm{Cl}(0.5 \mathrm{mM}$ nitrate $+1 \mathrm{mM}$ ammonium $)$. Other nutrient elements were supplied according to the Hogland medium (Hoagland and Snyder 1933). The experiment was repeated 3 times, at least 20 plants per inbred lines were treated each time, liquid medium was changed every $2 \mathrm{~d}$. Ten days after treatment, plants were harvested for phenotype observation such as fresh mass, dry mass (drying at $80{ }^{\circ} \mathrm{C}$ to constant mass), root and shoot length. Roots of inbred lines B73 (LN sensitive) and MBST (LN tolerance) were also harvested at 0, 2, 4, 6, 8, and $12 \mathrm{~h}$ after $\mathrm{LN}$ treatment and stored at $-80^{\circ} \mathrm{C}$ until use.

Bioinformatic analysis of genes related to $\mathbf{N}$ transport, $\mathbf{N}$ metabolism, $\mathbf{C}$ metabolism and some others: According to previous reports (Plett et al. 2010, 2016, Garnett et al. 2013, Gu et al. 2013, Léran et al. 2014, Zanin et al. 2014), database in MaideGDB website (https://www.maizegdb. org/), phytozome website (https://phytozome.jgi.doe. gov/pz/portal.html) and PLAZA https://bioinformatics. psb.ugent.be/plaza/versions/plaza_v4_monocots/, CDS sequences of ZmNRTs, ZmNPFs, ZmAMTs, ZmNLPs, $Z m N R, Z m N I R$ and carbon metabolism-related genes were searched. A 43 bp nitrate-responsive cis-elements (NREs) of ZmNIR (GRMZM2G079381) and AtNIR were used as references to identify potential NRE elements in the promoter $(-3000 \pm 1$ upstream the translation initiate site of ATG) of ZmNRT1s, ZmNRT2s and ZmNRT3s (LiseronMonfils et al. 2013, Konishi et al. 2014, Von Wittgenstein et al. 2014). Conserve sequences were found at http:// meme-suite.org/tools/meme and http://plantpan2.itps. ncku.edu.tw/ (Grant et al. 2011, Bailey et al. 2015, Chow et al. 2016). Some other genes which showed response to LN in other maize cultivars reported by Nazir et al. (2016) were choose to detect the expression in B73 and MBST plants.

Isolation of DNA and RNA, cDNA synthesis and plasmid extraction: Genomic DNA of B73 and MBST was extracted using a plant DNA extraction kit (Tiangen, Beijing, China) according to the manufacture's manual. The total RNA of each sample was isolated using a MiniBEST universal RNA extraction kit (TaKaRa, Dalian, China) and digested with RNase-free rDNase I (TaKaRa) according to the manufacturer's protocol. The DNA and RNA concentration and quality were evaluated using NanoDrop One (Thermo Fisher, Shanghai, China) and $1 \%(\mathrm{~m} / \mathrm{v})$ agarose gel. The cDNA was synthetized using PrimeScript $^{\mathrm{TM}}$ RT reagent with gDNA eraser (TaKaRa, Dalian, China). Plasmid was isolated using Plasmid Mini preparation kit (Beyotime, Shanghai, China).

Quantitative reverse transcription PCR: After cDNA synthesis, quantitative PCR was performed in an $A B I 7500$ real-time PCR system with 7500 software v2.0.4. A PCR $\operatorname{mix}\left(0.02 \mathrm{~cm}^{3}\right)$ was done by the $2 \times$ TB Green Premix Ex Taq II (TaKaRa, Dalian, China) user manual. Thermoamplification was performed in a 96-well plate (Applied Biosystems, Carlsbad, USA) following our previous procedure (Yu et al. 2015). Using actin as an internal control, expression of a target gene was compared to an internal gene (Chen et al. 2018). Results were obtained from three biological repeats (mixed together) and three technical repeats. Gene specific primers are listed in Table 2 Suppl.

Cloning promoter of $Z m N R T 3.1 B$ in B73 and MBST: According to sequences of B73, specific primers (Table 2 Suppl.) were designed to amplify -3338 to +1 regions of ZmNRT3.1B (pZmNRT3.1B) in B73 and MBST. Amplicons of PCR were cloned into the pGEM-T easy vector (Promega, Madison, WI, USA), positive clones were sequenced by General Biosystems (Chuzhou, China). The dNTP and PrimerSTAR GXL DNA polymerase were bought from TaKaRa, Dalian, China.

Measurement of total nitrogen, protein and sugar: Total content of nitrogen, proteins and sugars were determined. About $0.5 \mathrm{~g}$ dry material was digested with $18.4 \mathrm{M} \mathrm{H}_{2} \mathrm{SO}_{4}$, and the total nitrogen was measured by an automatic Kjeldahl apparatus (UDK 159, VELP Scientifica, Usmate, Italy). Total soluble protein was extracted according to the method described by Rocha and de Meis (1998). The concentration of protein was determined by using Protein Assay (Bio-Rad, Shanghai, China) at $595 \mathrm{~nm}$ (a PerkinElmer, Enspire 2300 Multilabel reader). One gramme of fresh maize tissue was homogenized in $10 \mathrm{~cm}^{3}$ of distilled water, and total sugar was measured at $620 \mathrm{~nm}$ by anthrone colorimetry (Koehler 1952). The experiments were repeated at least three times.

Statistical analysis of biomasses of roots and shoots, gene expressions and correlation among ZmNTRs and ZmNLPS were carried out using SSPS17. Significant differences between samples were tested using the Student's $t$-test. A correlation heatmaps of ZmNLPs and ZmNRTs were drawn by the $R$ language.

\section{Results and discussion}

We screened 30 inbred lines (Table 1 Suppl.) grown in hydroponic culture and found that B73, 2FACC, and many other inbred lines exhibited growth retardation, chlorosis of the base leaf tip, and light green coloration of the entire plant under LN conditions (Fig. 1 and Fig. 1 Suppl.). Further, length of shoot was decreased in B73 and fresh and dry masses in B73 and in 2FACC (Table 1). While root length was decreased MBST and N-PH-P, no other obvious phenotypic variation were found in MBST, 6M502, and N-PH-P under LN compared with plants growing under CK (Fig. 1 and Fig. 1 Suppl.). Hence, B73, 2FACC, and some other inbred lines are LN-sensitive lines, while, e.g., MBST, 6M502, and N-PH-P are LN-tolerant lines. Previous studies also have shown that LN-tolerant cultivars maintain their biomass under LN conditions (Gaudin et al. 2011, Garnett et al. 2015). In the present study, the primary roots (PR) of MBST plants grown under $\mathrm{LN}$ conditions were shorter than in plants grown under $\mathrm{CK}$ 
Table 1. Traits of five inbred lines under normal (CK) and low nitrogen (LN) conditions. Means \pm SEs, $n \geq 60$, * a significant difference between the same material under CK and LN conditions $(P<0.05, t$-test). B73 and 2FACC are LN-sensitive inbred lines, MBST, 6M502, and N-PH-P are LN tolerant lines.

\begin{tabular}{|c|c|c|c|c|c|c|c|c|}
\hline \multirow[t]{2}{*}{ Inbred lines } & \multicolumn{2}{|c|}{ Shoot length $[\mathrm{cm}]$} & \multicolumn{2}{|c|}{ Root length $[\mathrm{cm}]$} & \multicolumn{2}{|c|}{ Fresh mass [g plant $\left.{ }^{-1}\right]$} & \multicolumn{2}{|c|}{ Dry mass $\left[\mathrm{g} \mathrm{plant}^{-1}\right]$} \\
\hline & $\mathrm{CK}$ & $\mathrm{LN}$ & $\mathrm{CK}$ & $\mathrm{LN}$ & $\mathrm{CK}$ & $\mathrm{LN}$ & $\mathrm{CK}$ & $\mathrm{LN}$ \\
\hline B73 & $41.50 \pm 0.5$ & $32.65 \pm 4.65^{*}$ & $16.50 \pm 0.60$ & $17.95 \pm 0.35$ & $3.91 \pm 0.01$ & $2.64 \pm 0.55^{*}$ & $0.31 \pm 0.02$ & $0.21 \pm 0.06^{*}$ \\
\hline 2FACC & $40.88 \pm 2.38$ & $36.90 \pm 1.5$ & $27.80 \pm 1.85$ & $24.90 \pm 3.55$ & $4.87 \pm 0.56$ & $3.42 \pm 0.29 *$ & $0.31 \pm 0.03$ & $0.24 \pm 0.01 *$ \\
\hline MBST & $37.34 \pm 0.38$ & $38.78 \pm 2.44$ & $28.99 \pm 2.62$ & $23.03 \pm 1.93^{*}$ & $4.40 \pm 0.21$ & $4.05 \pm 0.58$ & $0.25 \pm 0.01$ & $0.25 \pm 0.03$ \\
\hline 6M502 & $38.73 \pm 2.51$ & $35.13 \pm 2.82$ & $18.43 \pm 2.16$ & $18.53 \pm 0.89$ & $4.08 \pm 0.81$ & $3.53 \pm 0.23$ & $0.23 \pm 0.05$ & $0.24 \pm 0.02$ \\
\hline N-PH-P & $34.42 \pm 2.94$ & $34.40 \pm 2.48$ & $24.68 \pm 3.36$ & $19.80 \pm 2.72 *$ & $3.15 \pm 0.03$ & $2.84 \pm 0.61$ & $0.21 \pm 0.01$ & $0.21 \pm 0.04$ \\
\hline
\end{tabular}

(Table 1, Fig. 1 Suppl.), the number and length of other types of roots (seminal roots and crown roots) increased. Under LN conditions, the primary roots of B73 were slightly longer than those grown under CK, but the density of laterals was unchanged and average number of SRs and CRs decreased by 10 - $15 \%$. Lynch (2013) proposed, that at different developmental stages root systems can adapt differently to the resources available in the soil. Early in seedling development, it is benefit to have a network of shallow roots to capture topsoil nutrients (Lynch 2013, Postma et al. 2014). When comparing the root systems of B73 and MBST plants, the network of shallow roots, such as CRs and SRs, was more extensive in of MBST than in B73 (Fig. 1 Suppl.). However, the deeper primary roots of B73 may beneficial for acquiring nutrients in deep soils. Based on these findings, we chose MBST and B73 to perform a comparative analysis of the transcriptions of N-related genes.

In the maize genome, $97 \mathrm{NRT} / \mathrm{PTF}$ members are divided into 8 clades according to their sequence similarity (Plett et al. 2010, 2016, Léran et al. 2014). In the present study, $Z m N R T$ genes were named following the nomenclature used by Plett et al. (2010). ZmNTR1s (10 members), ZmNTR2s (4 members), and ZmNTR3s (2 members) are homologs of AtNRT1s (LATS), AtNRT2s (HATS), and AtNRT3s (AtNRT3 functions as a chaperon of AtNRT2; Plett et al. 2010, 2016, Léran et al. 2014). Other genes were named following the nomenclature used by Léran et al. (2014).

Of the ten ZmNTR1s investigated, the expression of two genes (ZmNTR1.1C and ZmNTR1.4B) was not detected in the roots of both lines, while the others were detected in at least one line (Fig. 2). Of the expressed ZmNRT1s, $Z m N R T 1.1 A$ and 1.2 were highly expressed in both lines, while $Z m N R T 1.1 B$ and $1.1 D$ were moderately expressed. These four members accounted for more than $90 \%$ of the total ZmNTR1 expression. Under LN conditions, the expressions of all $7 \mathrm{ZmNTR} 1 \mathrm{~s}$ increased in the roots of B73

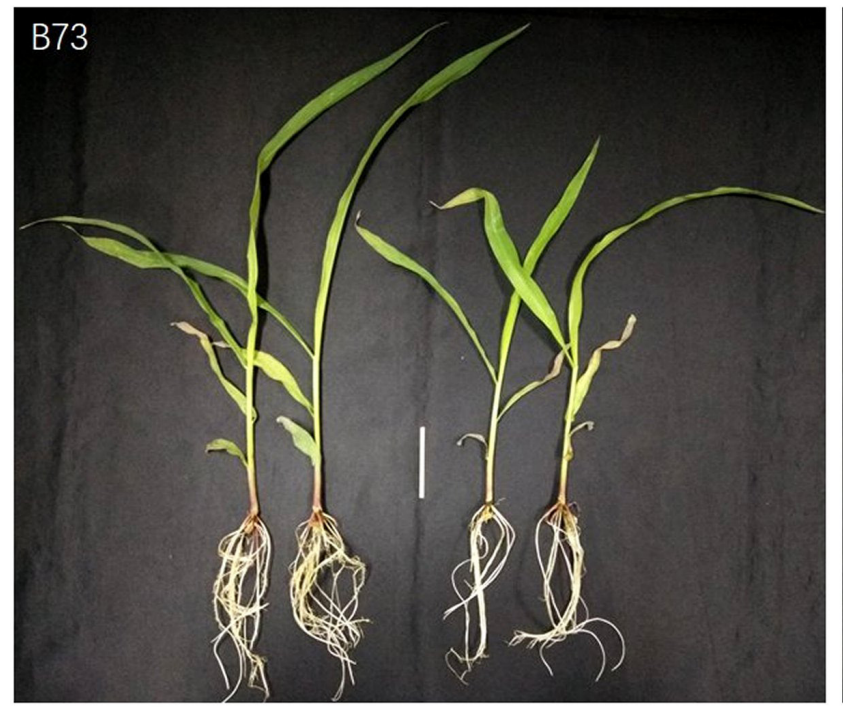

CK low nitrogen

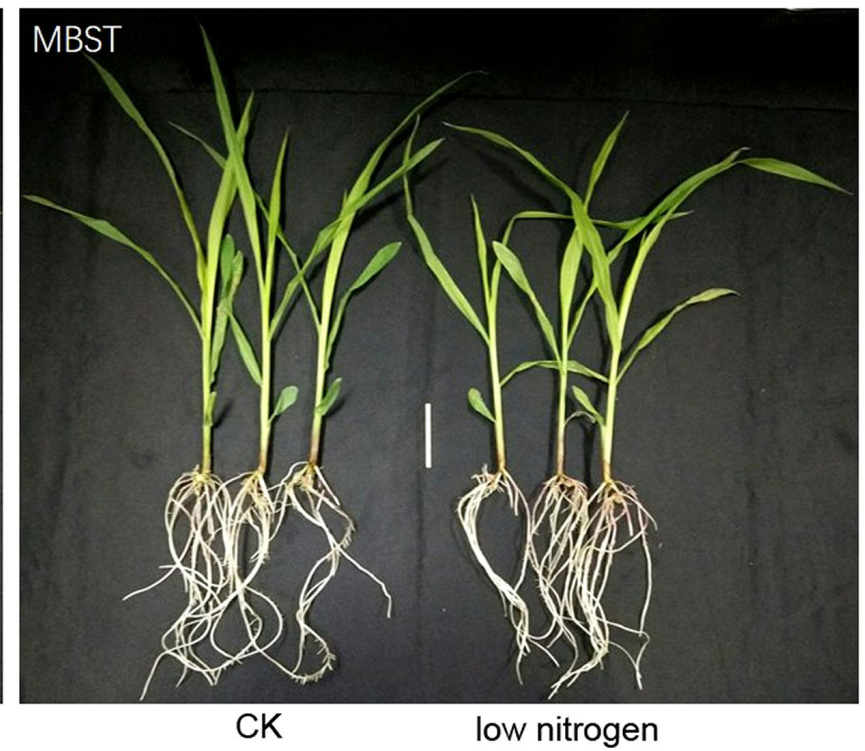

Fig. 1. The phenotype of B73 (sensitive) and MBST (tolerant) inbred lines under normal (CK) and low nitrogen (LN) conditions. CK- 6 mM nitrate $+1 \mathrm{mM}$ ammonium; LN - $0.5 \mathrm{mM}$ nitrate $+1 \mathrm{mM}$ ammonium. Plants were harvested after $10 \mathrm{~d}$ of LN treatment applied to 9-d-old seedlings; the bars are $5 \mathrm{~cm}$. Phenotypes of 6M502, N-PH-P, and 2FACC are shown in Fig. 1 Suppl. Data are not shown for other inbred lines. 


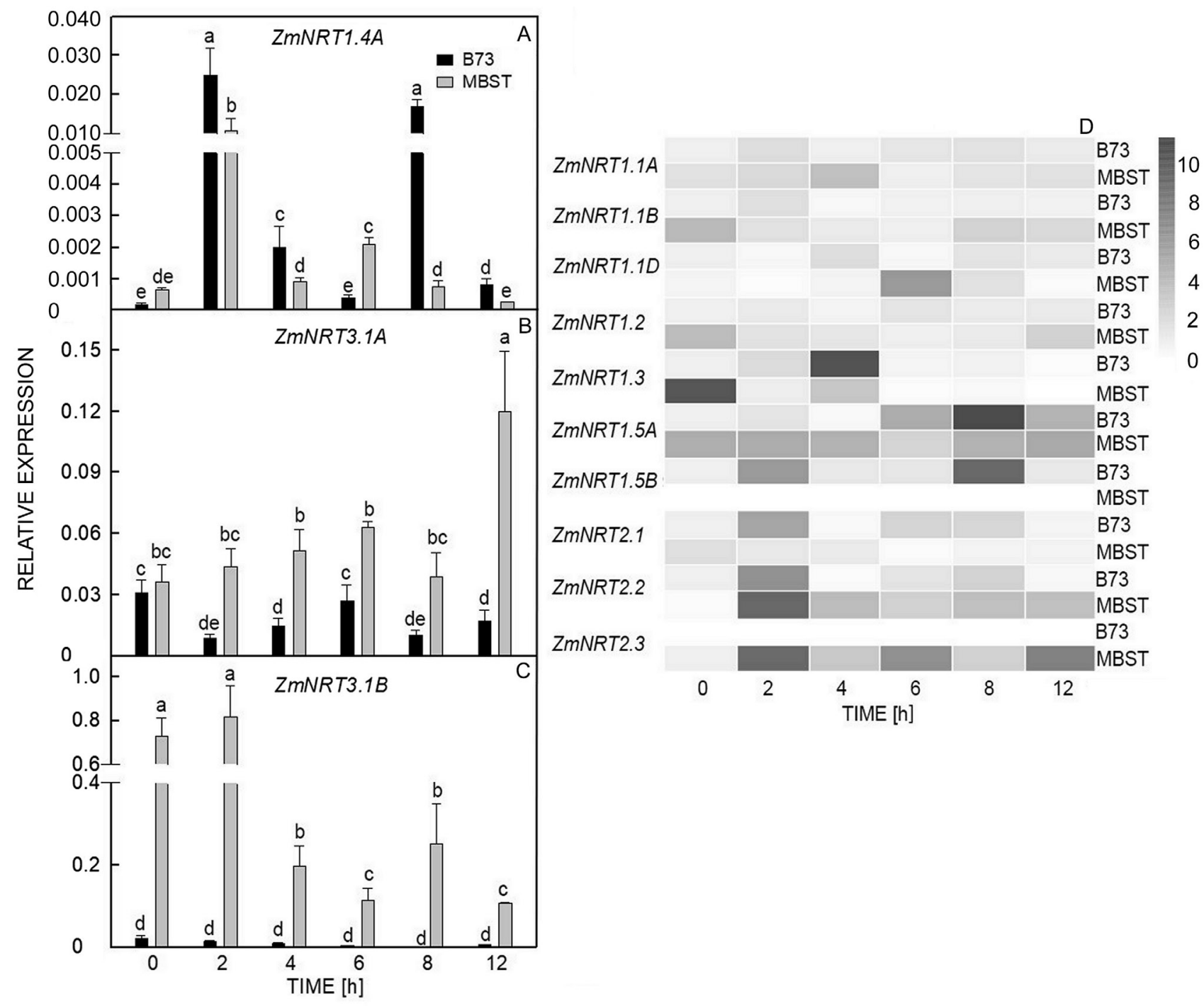

Fig. 2. Nitrate-dependent expression patterns of $Z m N R T s$. Maize plants were pre-cultured under $6 \mathrm{mM}$ nitrate and $1 \mathrm{mM}$ ammonium for $9 \mathrm{~d}$ before transfer to a $0.5 \mathrm{mM} \mathrm{NO}_{3}{ }^{-}$and $1 \mathrm{mM} \mathrm{NH}_{4}{ }^{+}$. Gene expressions were detected after low nitrogen treatments for $0,2,4,6,8$, or 12 h. $A$ - ZmNRT1.4A expressions in B73 and MBST at different time points; $B$ - ZmNRT3.1A expressions; $C$ - ZmNRT3.1B expressions. Gene expressions were normalized to actin expression (GRMZM2G126190). Means \pm SDs, $n=3$. Different letters indicate significant differences at $P<0.05$ (the Student's $t$-test). $D$ - The relative expression heatmap of ZmNRTs.

plants to a certain extent over a short period of time and then decreased. After $12 \mathrm{~h}$ of LN treatment, there were no significant differences compared with plants grown under CK (Fig. 2D). Although, the expressions of ZmNRT1.1A, $N R T 1.1 B$, and NRT1.2 in MBST plants decreased under LN conditions, the overall levels were still higher than those in B73 plants (Fig. 2D). Similar differences in expression patterns of $Z m N R T 1.4 A$ were detected between both lines (Fig. 2A). Our results were consistent with previous reports that the expression of most ZmNRT1s were unchanged or declined under long-term LN or nitrogen-deficient conditions (Wen et al. 2017). This may be because NRT1s are LATS, which are not the main transport proteins under LN conditions and therefore, it is expected that their expression will be reduced.

ZmNRT2.1 and 2.2 were the main ZmNRT2 (HATS) members expressed in the two lines. Their expression increased rapidly under LN conditions. ZmNRT2.2 was persistently induced in the MBST line, but its expression in B73 plants decreased after long-term treatment (Fig. 2D). Previous studies have shown that ZmNRT2.1 expression is induced by low nitrate input in a different maize lines, but it is not correlated with LN tolerance (Santi et al. 2003, Sorgonà et al. 2011). However, the induced expression of ZmNRT2.2 is positively correlated to genotypes with LN tolerance (Garnett et al. 2015). Our results were consistent with the above results, suggesting that ZmNRT2.2 is indeed the main transporter under LN conditions.

It is interesting to note that $Z m N R T 3.1 A$ and $Z m N R T 3.1 B$ were moderately expressed genes (about one order of magnitude lower than the internal control 


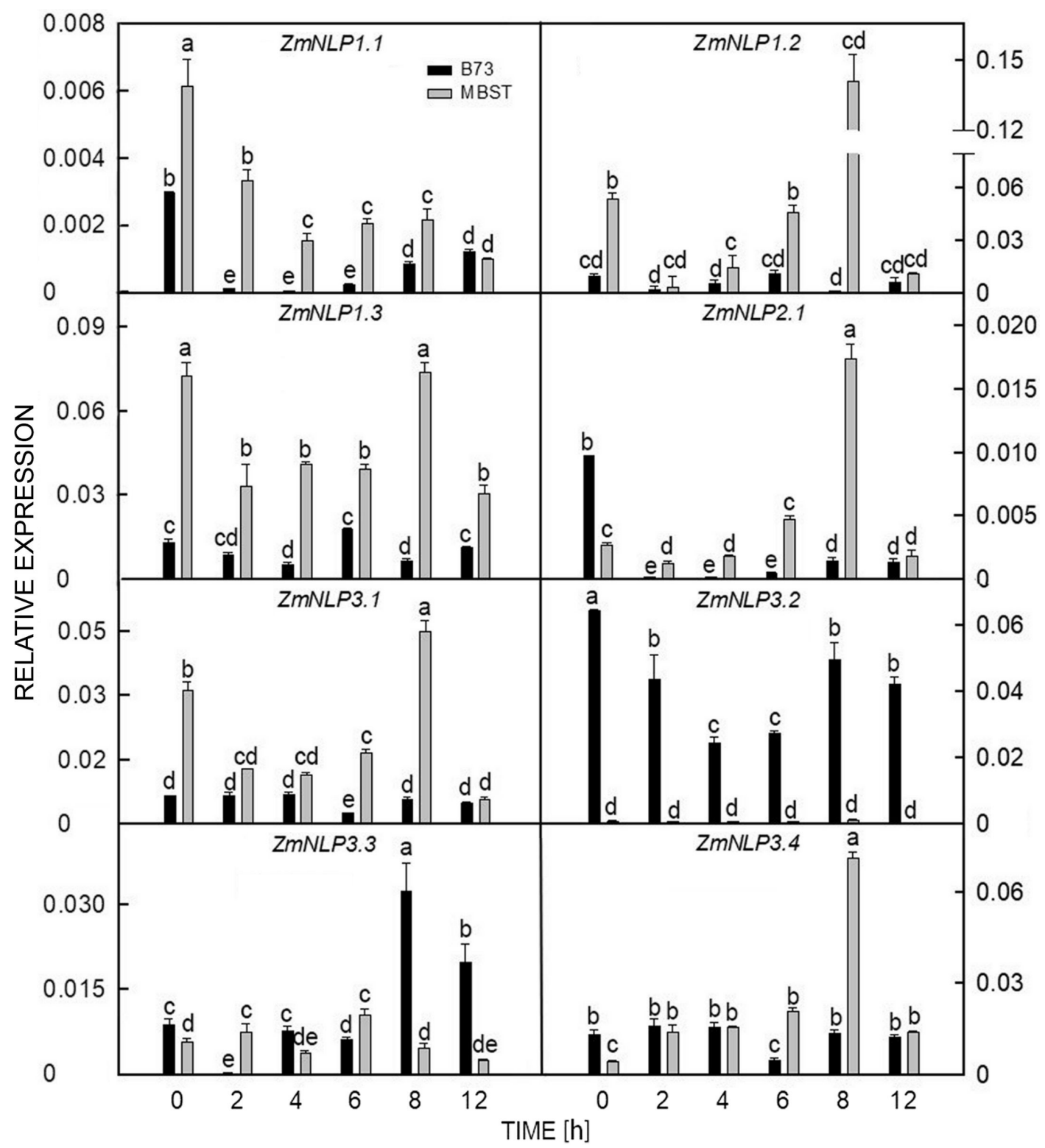

Fig. 3. Expression patterns of ZmNLPs under low nitrogen treatment for 0, 2, 4, 6, 8, or $12 \mathrm{~h}$. Expressions of ZmNLPs are shown as black columns for B73 and as gray columns for MBST. All expressions were normalized to actin (GRMZM2G126190) expression. Seedlings were pre-cultured under $6 \mathrm{mM}$ nitrate and $1 \mathrm{mM}$ ammonium for $9 \mathrm{~d}$ before transfer to a $0.5 \mathrm{mM} \mathrm{NO}_{3}{ }^{-}$and $1 \mathrm{mM} \mathrm{NH} 4^{+}$. Means $\pm \mathrm{SDs}$, $n=3$. Different letters indicate significant differences at $P<0.05$ (the Student's $t$-test).

actin) in B73 (Fig. 2B,C). These results are consistent with RNA-Seq data in the Expression Atlas (https://www. ebi.ac.uk/gxa/genes/) and the report by Garnett et al. (2015). However, NRT3.1B expression was significantly higher in the MBST line than in the B73 line (Fig. 2C). To determine the reason for this difference, we cloned the promoter (from -3338 to +1 ) of $Z m N R T 3.1 B$ in MBST, and investigated differences in the promoter between the two lines. Five single nucleotide polymorphisms (SNPs) and two small insertions/deletions were found between -3000 and -2000 (Fig. 2 Suppl.). These may be the cause of the transcriptional differences between the two lines, since cis-elements in the promoter recruit proteins that regulate gene expression. Further experiments are required to determine which polymorphism in the promoter leads to the variation in expression. NRT3s serve as chaperones for NRT2s and in Atnrt3 (nar2.1) mutant or knock-down lines of Arabidopsis and rice, NRT2s expression is also down-regulated (Okamoto et al. 2006, Yong et al. 2010, Yan et al. 2011). This may be why higher ZmNRT3.1s expression led to increased ZmNRT2.2 and 2.3 expressions in MBST. Further experiments are needed to identify the mechanism whereby ZmNRT3.1s promote the expression of ZmNRT2.2. Considering that all detected NRT2·NRT3s serve as HATS, specifically in nitrate influx in other plants, we speculate that higher expressions of ZmNRT2.2 and $Z m N R T 3.1 B$ may increase the efficiency of $\mathrm{NO}_{3}{ }^{-}$uptake in the MBST line. 
A

$\begin{array}{lr}\text { ZmNRT1.1A } & -2591 \\ \text { ZmNRT1.1B } & -378 \\ & -654 \\ & -2396 \\ \text { ZmNRT1.2 } & -2037 \\ \text { ZmNRT1.3 } & -1505 \\ \text { ZmNRT1.5A } & -93 \\ & -138 \\ \text { ZmNRT1.5B } & -3004 \\ & -1327 \\ \text { ZmNRT2.1 } & -253 \\ & -2399 \\ & -1852 \\ & -865 \\ \text { ZmNRT2.2 } & -656 \\ & -1239 \\ & -1364 \\ & -1449 \\ & -1493 \\ \text { ZmNRT2.5 } & -2580 \\ \text { ZmNRT3.1B } & -130 \\ & -288 \\ \text { ZmNIR } & -2099 \\ \text { AtNIR } & -114 \\ & -104\end{array}$

AGCTGGTAGCTAGCTCTTTCGAATGTGCAGGT CGATCCAGT GC ACGCCAAAAACGACCGTTCCTCGGGAGCCAGGCTCGT CGCGTG CGCTCACCAT TGACGCTTGGT GAGT CTTCTTCATCCCTTTGTC ATTGT GGCTTTCTCTCATTTGGT TGACAATAGAGCTCAGGGGC CCACTAGATAAGACTCTTCGTTTCATAGGTAGCT GCCAATAAT CCGTCGCT GT CGGCCCTT TCCTT GGCCT GGCCCCCACACGT GA CAGCTAGTAGCT GCTCTTGTGAT CGAGGATAT GGCAATGGT GG AGAACGAGGGAGGGGGTTGGGGCGATTTATAGAGCTCAGAATT GCTTTTGT TATGACCGTTGAATAAAATAGAATTTCCTAGATGA GAAGAGAAAGCT GGGAGCCGGAGGCCGGAGGGGGGCAGGGGCA AGCTT GACGACGACCCTTCAT GT CCAT GACACGCCAGAGCTCA CATCATTCGT TGGCTCTTCTTCCTCTGATTCATCATT GTCCTC CAACT CGCTCTT CAAT TTTTTCTAGGT CAAGGGT GCTACCCCT CGGCACACAACAAGT GT GGCGAGGCTTCAAGGTGGCATGCCGC TTCGCTGT TGCGGCCGTTCGACT GCGGGTACGTTACGCCTAGC AATAT GCATGTGGCCCTTAGGACTTATCAGAGCT GCAGCCGAC TGT GAACGCATGGCTGTTAATTAGT GTT TAGACT GAATGGAAT AAAATAAACT TGACTGTTGGTACGTACAACAGCCGTACACAGC TAGCT GT GTACGGCTGTTGTACGTACCAACAGTCAAGTTTATT CCACT TTACATGGCCGTTTACAT GGCGGCT GAATAT GGCCT TA GCGCGGT CGATGG- - - TTGGGGACGGCGAGGGGGCAACGTGGA TTTTGCAGTTTGACCCTTTCTTAGAAAAAAAATCACGTTTGGA TTTTGCAGTTTGACCCTTTCTTGGAAAAAAAATCACGTTTAGA CGCTGGT TCT TGGCTCTTGGGGAGTTCAAGGGGGCAGGCAGAC AGAAACAACT TGACCCTTTACATTGCTCAAGAGCTCATCTCTT

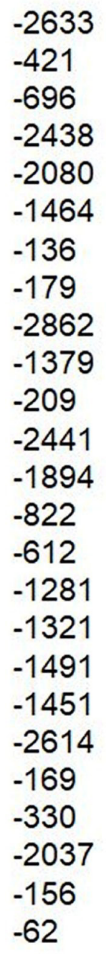

C

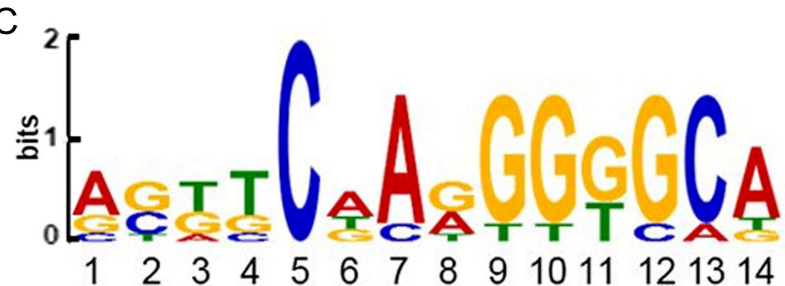

D
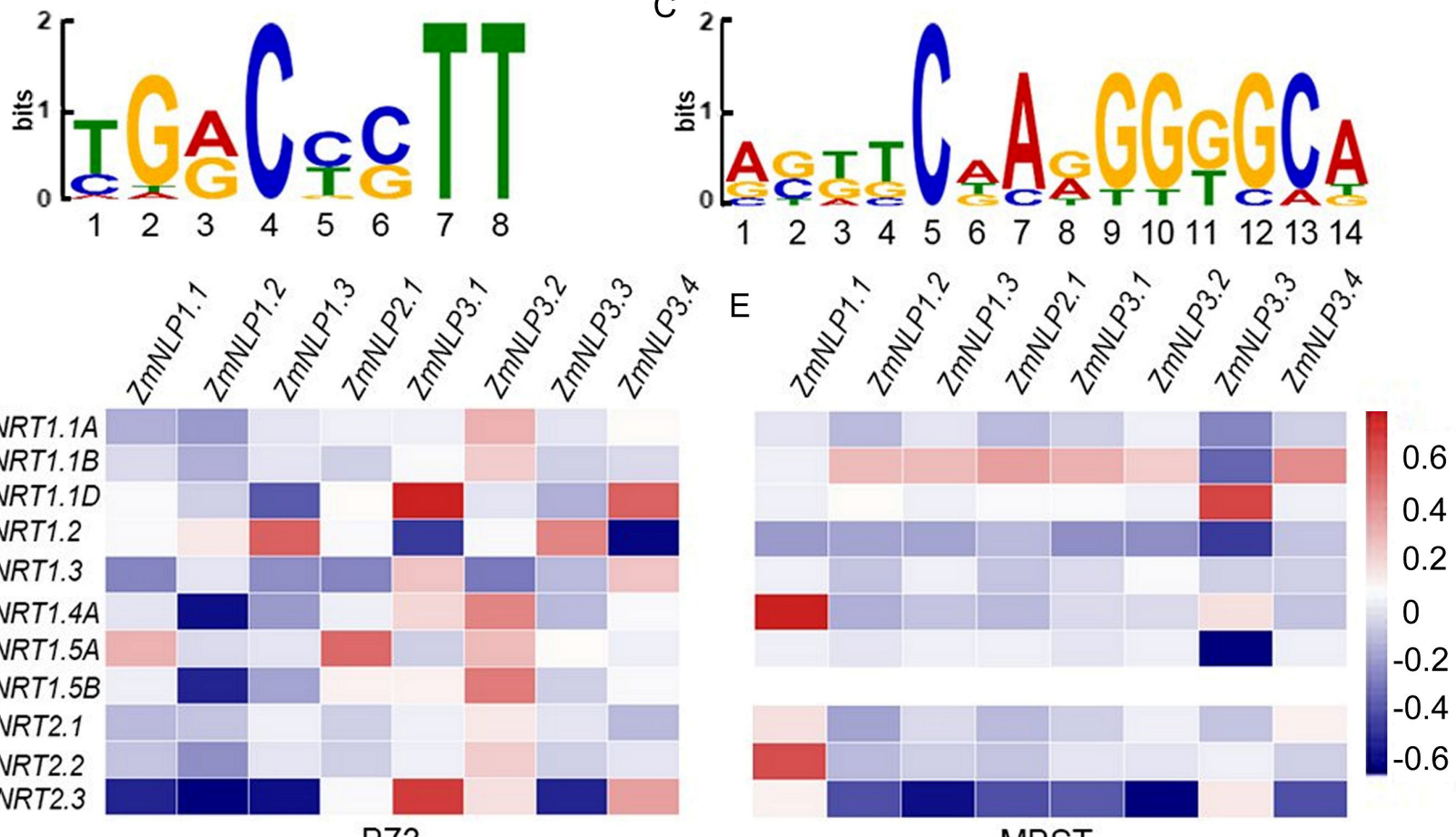

B73

MBST

Fig. 4. The ZmNLP negatively correlates with $Z m N R T s . A$ - nitrate-responsive cis-elements (NREs) in ZmNRT promoters. Yellow regions are NRE-A motifs and gray regions are NRE-B motifs. Numbers are upstream sites of ATG $(+1) . B$ and $C$ - Logos of NRE-A and -B motifs (http://meme-suite.org/tools/meme). The 43-bp nodule inception-like protein binding sites in Arabidopsis (AtNIR) and maize (ZmNIR) were used as a reference (Liseron-Monfils et al. 2013). $D$ and $E$ - The heatmap of the relationship between $Z m N L P s$ and ZmNRT mRNAs. Relative expressions of ZmNLPs and ZmNTRs were analyzed after 2, 4, 6, 8, or $12 \mathrm{~h}$ of low nitrogen treatment in inbred maize lines B73 and MBST. All expressions were normalized to actin (GRMZM2G126190) expression. Means \pm SDs, $n=3$. Positive correlations are shown in red and negative in blue. 
In addition to the HATS and LATS members mentioned above, there are other ZmNPF members in maize that may carry out transmembrane transport of oligopeptides, auxins, and sugars (Plett et al. 2010, Guan 2017, Li et al. 2017). Previous studies have shown that ZmNPF8.8 (ZmPTR1) is an oligopeptide transporter, expressed in the scutellum of germinating seeds and in the mature zone of roots (Tnani et al. 2013). The functions of other ZmNPFs have not yet been identified. In the present study, the expression of 44 members of the $Z m N P F$ family were detected (gene names as Léran et al. 2014). Of these, 14 were expressed in roots, but the remaining 30 were not expressed under our experimental conditions (Table 2 Suppl.). Among these $14 Z m N P F$ family members, $Z m N P F 7.2,8.8$, and 8.9 were highly expressed in the roots of B73 plants, whereas ZmNPF5.4, 7.2, 7.4, 8.8, and 8.9 were the main members expressed in the roots of MBST plants (Fig. 3 Suppl.). The expression of ZmNPF7.2 and 8.8 was down-regulated in the two inbred lines, suggesting that they responded to LN signals. From the expression patterns of the $Z m N P F s$, we can conclude that only a few of them may be involved in acquiring oligopeptides, auxins, and sugars in maize roots.

Studies in the model plant, Arabidopsis, have shown that AtNLPs are transcription activators when nitrate is resupplied after long term $\mathrm{N}$ deprivation (Castaings et al. 2009, Konishi and Yanagisawa 2013, 2014, Yan et al. 2016, Liu et al. 2017). Wang et al. (2018) also investigated the transcription profiles of $Z m N L P s$ under nitrate re-supply after prolonged $\mathrm{N}$ starvation. They showed that $Z m N L P 3.2$ is the most abundantly expressed $Z m N L P$ family member in different organs of maize, and that most of the $Z m N L P S$ members are not regulated by the nitrate resupply. However, the variation in expression of $Z m N L P s$ when maize is shifted from normal $\mathrm{N}$ to $\mathrm{LN}$ growth conditions is not known. Moreover, the relationship between $Z m N L P s$ and $Z m N R T s$ under these conditions is also unknown.

Under our experimental conditions, we found that the predominantly expressed gene members differed between the two inbred lines. In the B73 line, ZmNLP3.2 was the most abundantly expressed $Z m N L P$. Its expression was approximately $7 \%$ of the actin expression. However, in the MBST line, ZmNLP1.2 and 1.3 were the two most abundantly expressed $Z m N L P$ gene, with expressions similar to the expression of ZmNLP3.2 in B73. ZmNLPs were found to have different $\mathrm{LN}$ response patterns in the two lines. In the B73 line, with the exception of ZmNLP3.3, ZmLNP expression decreased slightly at first and was then gradually restored during $12 \mathrm{~h}$ of treatment. However, in the MBST line, ZmNLP levels demonstrated a down-up-down pattern during $12 \mathrm{~h}$ of LN treatment. The accumulation of $Z m N L P$ mRNA was less in both inbred lines under LN than in control plants. We conclude that sustained, long-term, LN conditions inhibit the expression of ZmNLPs.

AtNLPS induce downstream gene expression by binding to nitrate-responsive cis-elements (NREs). To uncover the relationship between $Z m N L P s$ and $Z m N R T s$, we investigated NREs in the promoters (from -3000 to +1 , where the A of the translation start site, ATG, is +1 ) of
ZmNRTs (Fig. 4). Several putative NREs were identified in the promoter region of ZmNRT2.1 and ZmNRT2.2. The left half motif of the NRE (named NRE-A in this study and highlighted in yellow in Fig. $4 A, B$ ) was more conservative than the right half (named NRE-B and shaded gray in Fig. $4 A, C$ ). Several NREs were identified in the promoters of ZmNRT2.1 and ZmNRT2.2, but no NREs were found in the promoters of ZmNRT.1.4A and ZmNRT2.3 (Fig. 4A).

Next, we investigated whether there is any correlation between the number of NRE motifs in the promoter and the expression of $Z m N R T s$. A correlation analysis of the expression of $Z m N L P s$ and $Z m N R T s$ showed a complicated relationship in the two inbred lines (Fig. $4 D, E$ ). In the B73 line, $Z m N L P s$ showed a negative relationship with ZmNRT2s (Fig. 4D), while in the MBST line, ZmNLP1.1 and $Z m N R T 2.1,2.2$ were positively correlated, but the other ZmNLPs were negatively correlated with $Z m N R T 2 s$ (Fig. 4E). Thus, it appears that $Z m N R T$ expression was not correlated with the number of NREs, which suggests that only a few of the NREs are functional in vivo.

The negative relationship found between $Z m N R T s$ and $Z m N L P S$ in the present study challenges previous studies that have shown that AtNLPS are positive regulators of AtNRT2s. Our results indicated that there may be other factors overcoming the influence of NLPs. The recent work of two groups in Arabidopsis help us explain our results (Yan et al. 2016, Maeda et al. 2018). The GAPAtype transcription factors, AtNIGT1/HRS1s, are brakes for AtNRT2.1 and 2.4 under high nitrate conditions. Importantly, the transcriptions of AtNIGT1/HRS1s are positively correlated with external nitrate concentrations and are also induced by AtNLPS. Hence, under limited nitrate availability, the content of AtNIGT1/HRS1s is low, whereas the downregulation of NLPs leads to a decrease in AtNIGT1/HRS1s. Therefore, it may be that that repressor effect of AtNIGT1/HRS1s outweighs the effect of NLPs under LN conditions. The results of the current research may also be explained by a model involving the antagonistic functions of NLPs and NIGT1/HRS1s. In other words, under LN conditions, the decreased content of ZmNIGT1/HRS 1s may lead to the enhanced expression of $Z m N R T s$.

AMTs are coded by a small gene family in plants, with 6, 12, and 8 members in Arabidopsis, rice, and maize genomes, respectively (Plett et al. 2010, Kiba and Krapp 2016). Based on data in MaizeGDB database, ZmAMT2A has alternative splicing forms, which lead to asymmetric amplification and therefore, we only detected the remaining 7 members. Altogether, 6 of the 7 genes detected were expressed in the roots of the two inbred lines. In B73, most of the detected transcripts were $Z m A M T 1 C$ and $2 C$, whereas in MBST, ZmAMT1C, 2C, and $2 D$ were predominantly expressed. With the exception of $Z m A M T 1 A$, the transcriptions of the ZmAMTs were higher in MBST than in B73 (Fig. 5). The results also showed that $Z m A M T 1 B$ and $1 C$ mRNA was up-regulated in MBST after $12 \mathrm{~h}$ of treatment, while mRNA of the others was down-regulated. However, in B73, ZmAMT1A and $Z m A M T 2 D$ expressions increased, while expressions of the other ZmAMTs were unchanged or decreased 


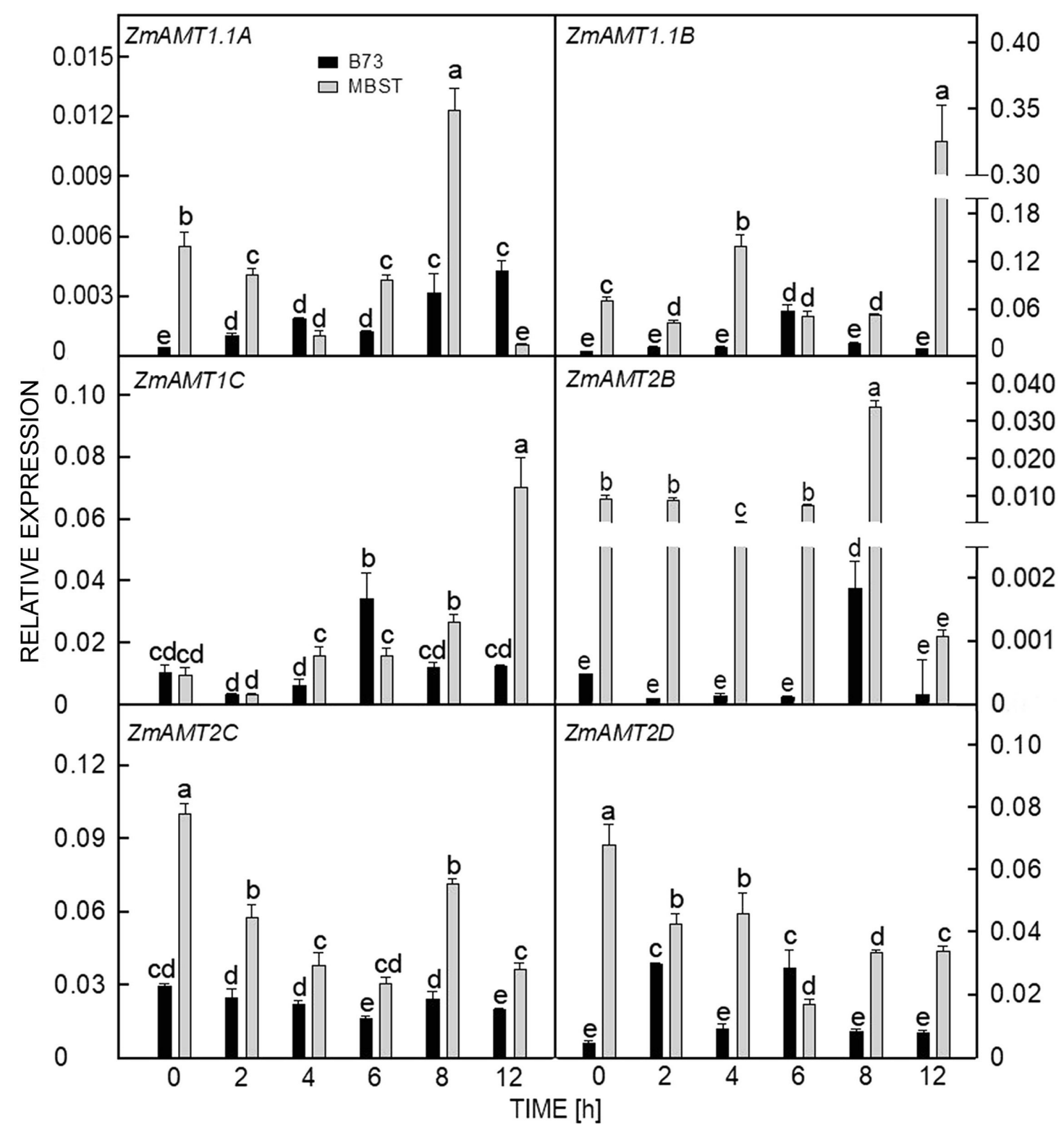

Fig. 5. Expressions of ZmAMTs under low nitrogen treatment for 0, 2, 4, 6, 8, or $12 \mathrm{~h}$. Expressions of ZmAMTs are shown by black columns for B73 and gray columns for MBST. All expressions were normalized to actin (GRMZM2G126190) expression. Means \pm SDs, $n=3$. Different letters indicate significant differences at $P<0.05$ (the Student's $t$-test).

(Fig. 5). Generally, our results showed that the expressions of ZmAMTs were relatively higher in the LN-tolerant line MBST, than in B73.

In the present study, only the nitrate content was changed in nutrient solution, but this nitrate could affect the abundance of ZmAMTs. The higher content of ZmAMT mRNA may lead to increased accumulation of ZmAMT protein, thus enhancing $\mathrm{NH}_{4}{ }^{+}$transport activity. ZmAMT1.1a and ZmAMT1.3 are the major high-affinity ammonium transporters and their expression is persistently induced by ammonium ( $\mathrm{Gu}$ et al. 2013). The results of this study suggested a higher efficiency of $\mathrm{NH}_{4}^{+}$capture in MBST than in B73, to partly compensate for limited nitrate availability and facilitate improved growth.
In addition to $\mathrm{NO}_{3}{ }^{-}$and $\mathrm{NH}_{4}{ }^{+}$transporter genes, we also measured the relative abundance of nine $\mathrm{N}$ assimilationrelated genes (Fig. 6). The results showed that four of these genes were expressed at moderate to high levels (Fig. 6B), while the others were expressed at low levels (Fig. 6A); seven of these nine genes were more highly expressed in MBST than in B73; and the content of ZmNIR and ZmGS $(G R M Z M 2 G 098290)$ mRNA increased significantly $(P<0.001, t$-test $)$ in MBST, but decreased in B73. As mentioned above, $\mathrm{NO}_{3}{ }^{-}$is converted to $\mathrm{NO}_{2}^{-}$by NR and then to $\mathrm{NH}_{4}{ }^{+}$by NIR. $\mathrm{NH}_{4}{ }^{+}$then serves as the substrate for GS and GOGAT enzymes, which catalyze Glu and Gln biosynthesis (Li et al. 2017). In MBST, the expression of these genes were higher than or at least similar to their 


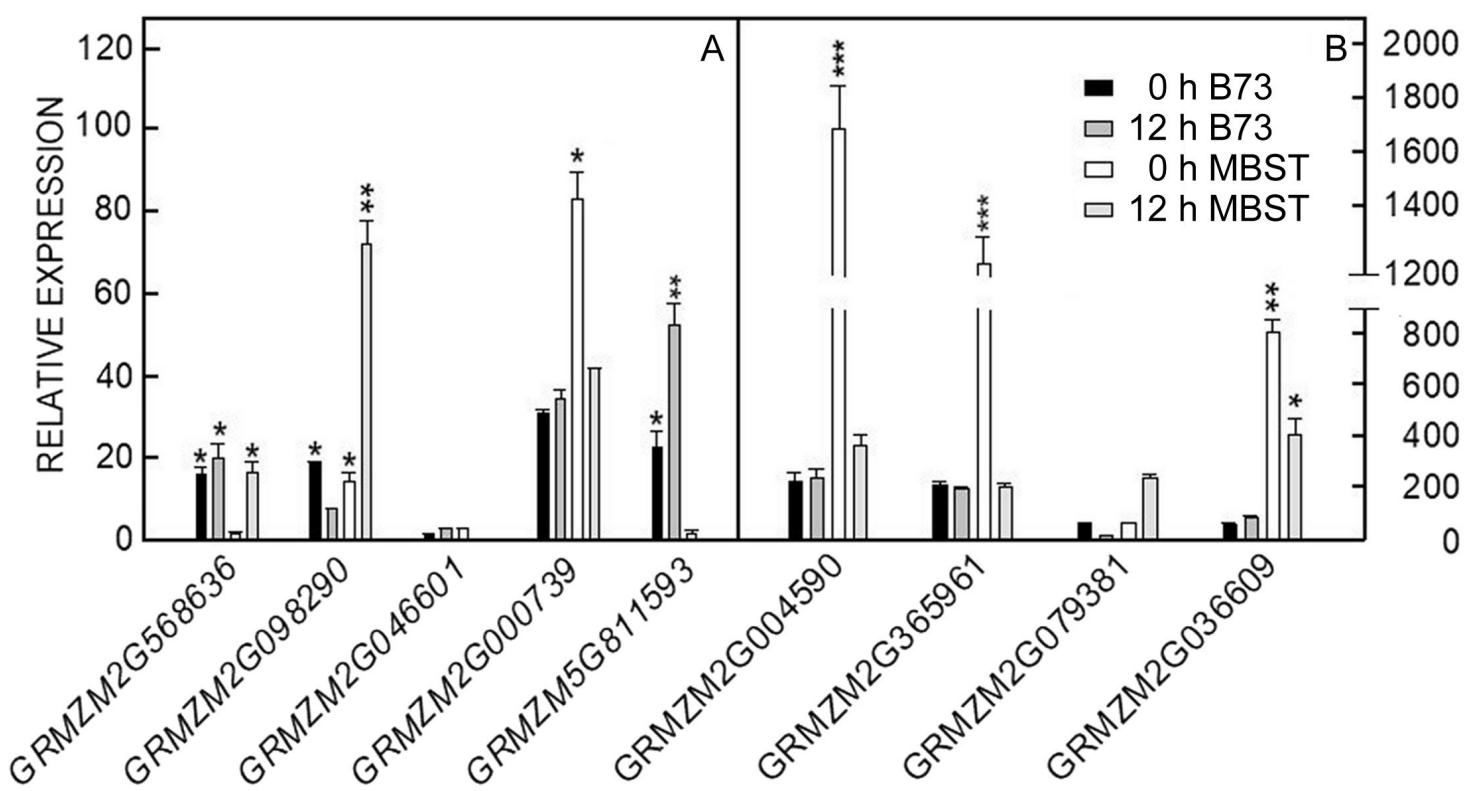

Fig. 6. Changes in expressions of $\mathrm{N}$ metabolism-related genes after $12 \mathrm{~h}$ of low nitrogen treatment. $A$ - Genes with a low expression; $B$ - genes with a high expression. GRMZM2G568636 - nitrate reductase, GRMZM2G098290 - glutamine synthetase, GRMZM2G046601 - glutamine synthetase, GRMZM2G000739 - urophorphyrin methylase 1, GRMZM5G811593 - peptide transporter, GRMZM2G004590 shikimate kinase 1); GRMZM2G365961 - arogenate dehydrogenase isoform 2, GRMZM2G079381 - nitrite reductase, and GRMZM2G036609 - ferredoxin-dependent glutamate synthase. All gene expressions were normalized to actin (GRMZM2G126190) expression. Means \pm SDs, $n=3$. Significant differences at $*-P<0.05$, ** $-P<0.01$, and $* * *-P<0.001$ (the Student's $t$-test).

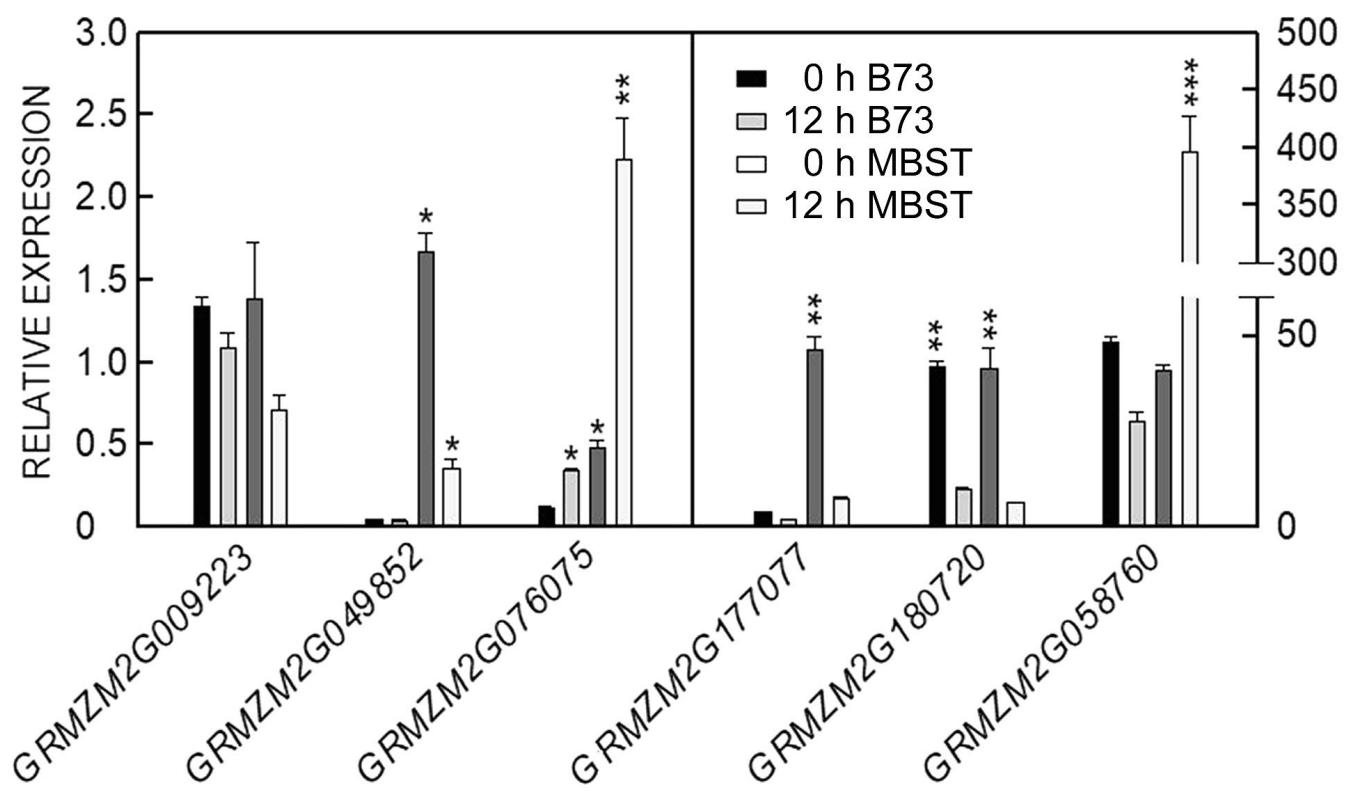

Fig. 7. Changes in the expressions of $\mathrm{C}$ metabolism-related genes and some other genes after $12 \mathrm{~h}$ of low nitrogen treatment. $A$ - Genes with a low expreion; $B$ - genes with a high expression. GRMZM2G009223 - glucose-6-phosphate translocator 2, GRMZM2G049852 - protein detoxification 49, GRMZM2G076075 - glucose-6-phosphate isomerase 1, GRMZM2G177077 - glucose-6-phosphate-1dehydrogenase, GRMZM2G180720 - glucose-6-phosphate translocator 2, and GRMZM2G058760 - ferredoxin-NADP reductase root isozyme 1. All gene expressions were normalized to actin (GRMZM2G126190). Means \pm SDs, $n=3$. Significant differences at * $-P<$ $0.05, * *-P<0.01$, and *** $-P<0.001$ (the Student's $t$-test).

expression in B73 after $12 \mathrm{~h}$ of LN treatment, suggesting that MBST has a higher capacity than B73 to assimilate $\mathrm{NO}_{3}{ }^{-}$under the conditions of our experiment.

Two of the four detected carbon metabolism-related genes were highly expressed (Fig. 7B). The mRNA content of ZmGPI (GRMZM2G076075) and ZmGPDH (GRMZM2G177077) were significantly different between the two lines $(P<0.05)$. In both lines, $Z m G P T s$ (GRMZM2G009223, GRMZM2G180720) had the same expression profile, being down-regulated by $12 \mathrm{~h}$ of 
LN treatment. Our results showed that the expression of all four genes tested were influenced by $\mathrm{N}$ concentration in nutrient solution.

The expression of other gene, such as GRMZM2G058760 (ZmFENR) was significantly upregulated after $\mathrm{LN}$ treatment $(P<0.001$, Fig. $7 B)$ in the MBST line. FENR plays a key role in regulating the relative amounts of cyclic and non-cyclic electron flow to meet the demands of the plant for ATP and reducing power (NADPH, Küchler et al. 2002). A higher ratio of $\mathrm{NADPH} / \mathrm{NADP}^{+}$means that the cell has ample energy for growth, which is especially important in rapid growth stages, such as in the maize seedling. The expression of GRMZM2G049852 increased dramatically in the MBST line after LN treatment. As a defense-related gene, it may confer tolerance of MBST to LN (Nazir et al. 2016).

Although, limited genes related to carbon or energy metabolism were detected in this study, it is well known that there is an interaction between nitrogen and carbon metabolism (Nunes-Nesi et al. 2010, Lynch 2013). Nazir et al. (2016) reported that most proteins fluctuate with nitrogen supply, which also affected carbon metabolism. In the present study, the content of total nitrogen and soluble proteins in B73 was lower than in MBST (Fig. 4 Supppl.), while content of soluble sugars was higher in B73 (Fig. 4 Suppl.). Saccharides are resources for protein, nucleic acid, and lipid biosynthesis and have complicated effects on plant development. Larger amounts of soluble sugars accumulated in B73 may reflect reduction of their use for biosynthesis of others compounds.

Recently, Li et al. (2018) reported that in high NUE rice cultivars or transgenic lines, the expressions of genes such as NRT1.1B, NRT2.3A, AMT1.1, NR, NIR, and GS are very high. Our results also showed that genes homologous to these in maize had rather high expressions in the LNresistant line MBST (Fig. 2, 5, and 6). From our results and those of previous reports (Guan 2017, Li et al. 2017, 2018), we conclude that plants may share common regulatory mechanisms for nitrogen utilization. The functions of some master genes, such as NLP and NIGT1/ HRS1, have not been fully elucidated in maize (Li et al. 2018, Kiba et al. 2018, Wang et al. 2018) and therefore, further research is needed to determine how these genes regulate nitrogen utilization and growth in maize.

\section{Conclusions}

In this study, we identified three LN-tolerant, inbred maize lines at the seedling stage. Based on the transcriptions of genes involved in $\mathrm{NO}_{3}{ }^{-}$and $\mathrm{NH}_{4}{ }^{+}$transport and assimilation and carbon metabolism, we conclude that the LN-tolerant line MBST have an increased capacity to transport external $\mathrm{NO}_{3}{ }^{-}$and $\mathrm{NH}_{4}{ }^{+}$into cells and to assimilate $\mathrm{N}$. Under LN conditions, the negative correlation between $Z m N L P s$ and ZmNRTs suggests there are other regulators antagonizing the ZmNLPs. Further research is necessary to uncover the relationship between ZmNRT2s and ZmNRT3s and to identify the mechanisms by which they regulate $\mathrm{N}$ signals under $\mathrm{LN}$ conditions.

\section{References}

Bailey, T.L., Johnson, J., Grant, C.E., Noble, W.S.: The MEME suite. - Nucl. Acids Res. 43 (Suppl.): W39-W49, 2015.

Cao, H., Qi, S., Sun, M., Li, Z., Yang, Y., Crawford, N.M., Wang, Y.: Overexpression of the maize ZmNLP6 and ZmNLP8 can complement the Arabidopsis nitrate regulatory mutant $n l p 7$ by restoring nitrate signaling and assimilation. - Front Plant Sci. 8: 1703, 2017.

Castaings, L., Camargo, A., Pocholle, D., Gaudon, V., Texier, Y., Boutet-Mercey, S., Taconnat, L., Renou, J.P., Daniel-Vedele, F., Fernandez, E., Meyer, C., Krapp, A.: The nodule inceptionlike protein 7 modulates nitrate sensing and metabolism in Arabidopsis. - Plant J. 57: 426-435, 2009.

Chen, Y., Cao, Y., Wang, L., Li, L., Yang, J., Zou, M.: Identification of MYB transcription factor genes and their expression during abiotic stresses in maize. - Biol. Plant. 62: 222-230, 2018.

Chow, C.N., Zheng, H.Q., Wu, N.Y., Chien, C.H., Huang, H.D., Lee, T.Y., Chiang-Hsieh, Y.F., Hou, P.F., Yang, T.Y., Chang, W.C.: Plant PAN 2.0: an update of plant promoter analysis navigator for reconstructing transcriptional regulatory networks in plants. - Nucl.Acids Res. 44 (Suppl.): D1154-D1160, 2016.

Garnett, T., Conn, V., Plett, D., Conn, S., Zanghellini, J., Mackenzie, N., Enju, A., Francis, K., Holtham, L., Roessner, U., Boughton, B., Bacic, A., Shirley, N., Rafalski, A., Dhugga, K., Tester, M., Kaiser, B.N.: The response of the maize nitrate transport system to nitrogen demand and supply across the lifecycle. - New Phytol. 198: 82-94, 2013.

Garnett, T., Plett, D., Conn, V., Conn, S., Rabie, H., Rafalski, J.A., Dhugga, K., Tester, M.A., Kaiser, B.N.: Variation for N Uptake system in maize: genotypic response to $\mathrm{N}$ supply. Front Plant Sci. 6: 936, 2015.

Gaudin, A.C., McClymont, S.A., Holmes, B.M., Lyons, E., Raizada, M.N.: Novel temporal, fine-scale and growth variation phenotypes in roots of adult-stage maize (Zea mays L.) in response to low nitrogen stress. - Plant Cell Environ. 34: 2122-2137, 2011.

Grant, C.E., Bailey, T.L., Noble, W.S.: FIMO: scanning for occurrences of a given motif. - Bioinformatics 27: 1017-1018, 2011.

Gu, R., Duan, F., An, X., Zhang, F., Von Wirén, N., Yuan, L.: Characterization of AMT-mediated high-affinity ammonium uptake in roots of maize (Zea mays L.). - Plant Cell Physiol. 54: 1515-1524, 2013.

Guan, P.Z.: Dancing with hormones: a current perspective of nitrate signaling and regulation in Arabidopsis. - Front Plant Sci. 8: 1697, 2017.

Han, J., Wang, L., Zheng, H., Pan, X., Li, H., Chen, F., Li, X.: ZD958 is a low-nitrogen-efficient maize hybrid at the seedling stage among five maize and two teosinte lines. - Planta 242: 935-949, 2015.

Hoagland, D.R., Snyder, W.C.: Nutrition of strawberry plant under controlled conditions. - Proc. Am. Soc. hort. Sci. 30: 288-294, 1933.

Kiba, T., Inaba, J, Kudo, T., Ueda, N., Konishi, M., Mitsuda, N., Takiguchi, Y., Kondou, Y., Yoshizumi, T., Ohme-Takagi, M., Matsui, M., Yano, K., Yanagisawa, S., Sakakibara, H.: Repression of nitrogen starvation responses by members of the Arabidopsis GARP-type transcription factor NIGT1/ HRS1 subfamily. - Plant Cell 30: 925-945, 2018.

Kiba, T., Krapp, A.: Plant nitrogen acquisition under low availability: regulation of uptake and root architecture. - Plant Cell Physiol. 57: 707-714, 2016.

Koehler, L.H.: Differentiation of carbohydrates by anthrone 
reaction rate and color intensity. - Anal. Chem. 24: 15761579, 1952.

Konishi, M., Yanagisawa, S.: Emergence of a new step towards understanding the molecular mechanisms underlying nitrateregulated gene expression. - J. exp. Bot. 65: 5589-5600, 2014.

Konishi M., Yanagisawa S: Arabidopsis NIN-like transcription factors have a central role in nitrate signaling. - Nat Commun. 4: 1617, 2013.

Küchler, M., Decker, S., Hörmann, F., Soll, J., Heins, L.: Protein import into chloroplasts involves redox-regulated proteins. EMBO J. 21: 6136-6145, 2002

Laugier, E., Bouguyon, E., Mauries, A., Tillard, P., Gojon, A., Lejay, L.: Regulation of high-affinity nitrate uptake in roots of Arabidopsis depends predominantly on posttranscriptional control of the NRT2.1/NAR2.1 transport system. - Plant Physiol. 158: 1067-1078, 2012.

Léran, S., Varala, K K., Boyer, J.C., Chiurazzi, M., Crawford, N., Daniel-Vedele, F., David, L., Dickstein, R., Fernandez, E., Forde, B., Gassmann, W., Geiger, D., Gojon, A., Gong, J.M., Halkier, B.A., Harris, J.M., Hedrich, R., Limami, A.M., Rentsch, D., Seo, M., Tsay, Y.F., Zhang, M., Coruzzi, G., Lacombe, B.: A unified nomenclature of NITRATE TRANSPORTER 1/PEPTIDE TRANSPORTER family members in plants. - Trends Plant Sci. 19: 5-9, 2014.

Li, H., Hu, B., Chu, C.C.: Nitrogen use efficiency in crops: lessons from Arabidopsis and rice. - J. exp. Bot. 68: 24772488, 2017.

Li, S., Tian, Y., Wu, K., Ye, Y., Yu, J., Zhang, J., Liu, Q., Hu, M., Li, H., Tong, Y., Harberd, N.P., Fu, X: Modulating plant growth-metabolism coordination for sustainable agriculture. Nature 560: 595-600, 2018.

Liseron-Monfils, C., Bi, Y.M., Downs, G.S., Wu, W., Signorelli, T., Lu, G., Chen, X., Bondo, E., Zhu, T, Lukens, L.N., Colasanti, J., Rothstein, S.J., Raizada, M.N.: Nitrogen transporter and assimilation genes exhibit developmental stage-selective expression in maize (Zea mays L.) associated with distinct $c i s$-acting promoter motifs. - Plant Signal Behav. 8: e26056, 2013.

Liu, G.W., Sun, A.L., Li, D.Q., Athman, A., Gilliham, M., Liu, L.H.: Molecular identification and functional analysis of a maize (Zea mays) DUR3 homolog that transports urea with high affinity. - Planta 241: 861-874, 2015.

Liu, K.H., Niu, Y., Konishi, M., Wu, Y., Du, H., Sun Chung, H., Li, L., Boudsocq, M., McCormack, M., Maekawa, S., Ishida, T., Zhang, C., Shokat, K., Yanagisawa, S., Sheen, J: Discovery of nitrate-CPK-NLP signalling in central nutrientgrowth networks. - Nature 545: 311-316, 2017.

Liu, K.H., Tsay, Y.F.: Switching between the two action modes of the dual-affinity nitrate transporter CHL1 by phosphorylation. - EMBO J. 22: 1005-1013, 2003.

Lv, Y., Liang, Z., Ge, M., Qi, W., Zhang, T., Lin, F., Peng, Z., Zhao, H.: Genome-wide identification and functional prediction of nitrogen-responsive intergenic and intronic long non-coding RNAs in maize (Zea mays L.). - BMC Genomics 17: 350, 2016.

Lynch, J.P.: Steep, cheap and deep: an ideotype to optimize water and $\mathrm{N}$ acquisition by maize root systems. - Ann. Bot. 112: 347-357, 2013

Maeda, Y., Konishi, M., Kiba, T., Sakuraba, Y., Sawaki, N., Kurai, T., Ueda, Y., Sakakibara, H., Yanagisawa, S.: A NIGT1centred transcriptional cascade regulates nitrate signalling and incorporates phosphorus starvation signals in Arabidopsis. Nat. Commun. 9: 1376, 2018.

Medici, A., Krouk, G.: The primary nitrate response: a multifaceted signalling pathway. - J. exp. Bot. 65: 5567-5576, 2014.
Nazir, M., Pandey, R., Siddiqi, T.O., Ibrahim, M.M., Qureshi, M.I., Abraham, G., Vengavasi, K., Ahmad, A.: Nitrogendeficiency stress induces protein expression differentially in low-N tolerant and low-N sensitive maize genotypes. - Front. Plant Sci. 7: 298, 2016.

Nunes-Nesi, A., Fernie, A.R., Stitt, M.: Metabolic and signaling aspects underpinning the regulation of plant carbon nitrogen interactions. - Mol. Plants 3: 973-996, 2010.

Okamoto, M., Kumar, A., Li, W., Wang, Y., Siddiqi, M.Y., Crawford, N.M., Glass, A.D.: High-affinity nitrate transport in roots of Arabidopsis depends on expression of the NAR2like gene AtNRT3.1. - Plant Physiol. 140: 1036-1046, 2006.

Plett, D., Baumann, U., Schreiber, A.W., Holtham, L., Kalashyan, E., Toubia, J., Nau, J., Beatty, M., Rafalski, A., Dhugga, K.S., Tester, M., Garnett, T., Kaiser, B.N.: Maize maintains growth in response to decreased nitrate supply through a highly dynamic and developmental stage-specific transcriptional response. - Plant Biotechnol. J. 14: 342-353, 2016.

Plett, D., Toubia, J., Garnett, T., Tester, M, Kaiser, B.N., Baumann, U.: Dichotomy in the NRT gene families of dicots and grass species. - PLoS ONE 5: e15289, 2010.

Postma, J.A., Dathe, A., Lynch, J.P.: The optimal lateral root branching density for maize depends on nitrogen and phosphorus availability. - Plant Physiol. 166: 590-602, 2014.

Riechmann, J.L., Heard, J., Martin, G., Reuber, L., Jiang, C., Keddie, J., Adam, L., Pineda, O., Ratcliffe, O.J., Samaha, R.R., Creelman, R., Pilgrim, M., Broun, P., Zhang, J.Z., Ghandehari, D., Sherman, B.K., Yu, G.: Arabidopsis transcription factors: genome-wide comparative analysis among eukaryotes. Science 290: 2105-2110, 2000.

Rocha, F.A., de Meis, L.: Reversibility of $\mathrm{H}^{+}$-ATPase and $\mathrm{H}^{+}$pyrophosphatase in tonoplast vesicles from maize coleoptiles and seeds. - Plant Physiol. 116:1487-1495, 1998.

Santi, S., Locci, G, Monte, R., Pinton, R, Varanini, Z: Induction of nitrate uptake in maize roots: expression of a putative highaffinity nitrate transporter and plasma membrane $\mathrm{H}^{+}$-ATPase isoforms. - J. exp. Bot. 54: 1851-1864, 2003.

Shen, L.X., Wang, P.: [Research progress of nitrogen absorption and utilization efficiency of different maize genotypes.] - J. Maize Sci. 24: 50-55, 2016. [In Chin.]

Sorgonà, A., Lupini, A., Mercati, F., Di Dio, L., Sunseri, F., Abenavoli, M.R.: Nitrate uptake along the maize primary root: an integrated physiological and molecular approach. Plant Cell Environ. 34: 1127-1140, 2011.

Tnani, H., López-Ribera, I., García-Muniz, N., Vicient, C.M.: ZmPTR1, a maize peptide transporter expressed in the epithelial cells of the scutellum during germination. - Plant Sci. 207: 140-147, 2013.

Von Wittgenstein, N.J., Le, C.H., Hawkins, B.J., Ehlting, J.: Evolutionary classification of ammonium, nitrate, and peptide transporters in land plants. - BMC Evol. Biol. 14: 11, 2014.

Wang, Y, Mi, G, Chen, F, Zhang, J., Zhang, F: Response of root morphology to nitrate supply and its contribution to nitrogen accumulation in maize. - J Plant Nutr. 27: 2189-2202, 2005.

Wang, Z., Zhang, L., Sun, C., Gu, R., Mi, G., Yuan, L.: Phylogenetic, expression and functional characterizations of the maize NLP transcription factor family reveal a role in nitrate assimilation and signaling. - Physiol Plant 163: 269$281,2018$.

Wen, Z., Tyerman, S.D., Dechorgnat, J., Ovchinnikova, E., Dhugga, K.S., Kaiser, B.N.: Maize NPF6 proteins are homologs of Arabidopsis CHL1 that are selective for both nitrate and chloride. - Plant Cell 29: 2581-2596, 2017.

Yan, D., Easwaran, V, Chau, V, Okamoto, M., Ierullo, M., Kimura, M., Endo, A., Yano, R., Pasha, A, Gong, Y., Bi, Y.M., Provart, N., Guttman, D D., Krapp, A., Rothstein, S.J., Nambara, E E.: 
NIN-like protein 8 is a master regulator of nitrate-promoted seed germination in Arabidopsis. - Nat. Commun. 7: 13179, 2016.

Yan, M., Fan, X., Feng, H., Miller, A.J., Shen, Q., Xu, G.: Rice OsNAR2.1 interacts with OsNRT2.1, OsNRT2.2 and OsNRT2.3a nitrate transporters to provide uptake over high and low concentration ranges. - Plant Cell Environ. 34: 13601372, 2011.

Yong, Z., Kotur, Z., Glass, A.D.: Characterization of an intact two-component high-affinity nitrate transporter from
Arabidopsis roots. - Plant J. 63: 739-748, 2010.

Yu, C., Liu, X., Zhang, Q., He, X., Huai, W., Wang, B., Cao, Y., Zhou, R.: Molecular genetic analysis of phosphomannomutase genes in Triticum monococcum. - Crop J. 3: 29-36, 2015.

Zanin, L., Tomasi, N., Wirdnam, C., Meier, S., Komarova, N.Y., Mimmo Cesco, T., S., Rentsch, D., Pinton, R.: Isolation and functional characterization of a high affinity urea transporter from roots of Zea mays. - BMC Plant Biol. 14: 222, 2014. 